\title{
Robot Assisted Smile Recovery
}

\author{
Dushyantha Jayatilake, Anna Gruebler, and Kenji Suzuki \\ University of Tsukuba \\ Japan
}

\section{Introduction}

\subsection{Facial Expressions}

Facial expressions play a significant role in social information exchange and the physical and psychological makeup of a person because they are an essential method for non-verbal communication. Through facial expressions, human beings can show emotions, moods and information about their character.

Happiness, sadness, fear, surprise, disgust, and anger, are typically identified by psychologists as basic emotions with their corresponding characteristic facial expressions (Wang and Ahuja; 2003). Further, (Batty and Taylor; 2003) reported that humans have a very fast processing speed when it comes to identifying these six expressions and noted that positive expressions (e.g. happiness, surprise) are identified faster than the negative expressions (e.g. sadness, disgust).

Human beings share common characteristics in the way they express emotions through facial expressions which are independent from nationality, ethnicity, age or sex. It has been recorded that the ability to recognize the corresponding emotion in a facial expression is innate and is present very early, possibly form birth (Mandler et al.; 1997). However there is also evidence that universal expressions might be modified in social situations to create the impression of culture-specific facial expression of emotions. For example, (Ekman; 1992) noted that when an authority figure was present, the Japanese masked negative expressions with the semblance of a smile more than the Americans. If, because of accident or illness, a person looses the ability to make facial expressions this makes the face seem emotionless and leads to physical and psychological hardships.

\subsection{Facial Paralysis}

The medical term "Paralysis" is defined as the complete loss of muscle function of one or more muscle groups. Facial paralysis is the total loss of voluntary muscle movement of one or both sides of the face. It can happen to anyone of any age. Paralysis often includes loss of feeling in the affected area, and is mostly caused by damage to the nervous system or brain. The paralysis can be short term which is between a few minutes and a few hours, long term which is usually at most about 6-7 months but sometimes registering even 4-5 years, or permanent (Byrne; 2004; Garanhani et al.; 2007). The House classification system describes 7 grades of facial paralysis (6 according to (Beck and Hall; 2001)) form Normal to Total, where the latter is the complete facial paralysis with no tone (Quinn and Jr.; 1996).

Facial nerve paralysis is a fairly common issue that involves the paralysis of any parts stimulated by the facial nerve. Due to the lengthy and relatively convoluted nature of the pathway 


\begin{tabular}{c|c}
\hline \hline Bell's Palsy & $0.5 / 1000$ per year \\
\hline recurrence rate & $7.0 \%$ \\
\hline lifetime prevalence & $0.64-2.0 \%$ \\
\hline
\end{tabular}

Table 1. Bell's Palsy statistics

of the facial nerve, several situations can result in facial nerve paralysis. The most common is Bell's palsy. As table 1 shows, the recorded cases of Bell's Palsy are 0.5 per 1000 persons per year with a recurrence rate of $7 \%$ and a lifetime prevalence of 6.4 to 20 per 1000 . The occurrences show an equal male to female ratio however it is 3.3 times greater among pregnant females (Quinn and Jr.; 1996). About $84 \%$ of the Bell's Palsy patients said to have had a spontaneous recovery which is likely to occur within the first three weeks. However the remaining $16 \%$ show only moderate to poor recovery. Facial paralysis due to a brain tumor generally develops slowly and causes headaches, seizures, or hearing loss. Facial paralysis, although not life threatening, can cause severe distraction in many ways. Physically, it makes the patient less responsive as well as having difficulties in eating, drinking and talking due to the inability to purse the lips.

Apart from the loss of volitional facial muscle motion, facial paralysis has another major consequence, which is the loss of baseline muscle tone. This causes the changes in facial appearance, such as drooping of the ipsilateral face and and deviation of the nose to the contralateral side (Pensak; 2001). The face is such a salient feature of a person that such a facial disfigurement can result in severe social and vocational handicap. A paralysis or weakness of even only one side of the face can be an alarming and depressing event in the patient's life.

\subsection{Current Medical Treatments and Rehabilitation for facial paralysis}

The current treatment methods for facial paralysis can be divided into three main categories: physical therapy, medical therapy, and surgery. Physiotherapy, facial exercises and massage are used to treat all types of facial paralysis. Physical therapy uses facial neuromuscular retraining, which is based on selective motor training techniques to optimize the motor control of the facial muscles.

There have been reports on the use of facial electromyography (EMG) to analyze emotions through facial expressions. It has been stated that because of its high temporal resolution, facial EMG is well suited for measuring emotions, which have rapid onset and short durations (Harrigan et al.; 2005).Along those lines, visual and bio-feedback through specific mirror exercises and surface electromyography (sEMG) based augmented sensory information is used to enhance neural adaptation and learning. It has been stated that sEMG based treatments allow the therapist to quantify the muscle activity patterns, resulting in more effective and faster treatment. Based on the study on training of nasal muscles, (Vaiman et al.; 2005) stated that Electromyography-recorded amplitude of muscle tension of the nasal muscles significantly increased in all the patients considered in the study. (Sugimoto1 et al.; 2007) in their article on autogenic training explained how sEMG can be used and data can be processed to improve treatments. Paralysis due to a compromised immune system, for instance, bacterial external ear infection, is often treated by using intravenous antibiotics. Bell's palsy treatments may involve the use of steroids and anti-viral drugs. The prime target of rehabilitation would be to recover the normal facial tone and function.

In the event of an identified break in the facial nerve, repairing and grafting are performed to reestablish the connection. The hypoglossal to facial nerve transfer reestablishes neuronal im- 
pulses to the facial muscles and supplies a baseline resting tone. However patients regaining the ability to show any spontaneous or emotive expression are said to be very rare with this method (Pensak; 2001).

Cross facial nerve grafting is the method of connecting the facial nerve of the paralyzed with the facial nerve of the healthy side by using a nerve obtained from the patient himself. This method said to produce symmetrical faces and since the grafting nerve is obtained from the same patient it eliminates the problems associated with rejection by the body. It is also worthwhile noting that to achieve the best results with facial reanimation techniques it is usually required to perform nerve repair or a combination of techniques that will lead to the strongest facial movement as soon after the injury as possible (May and Schaitkin; 2003).

\subsection{Smile Recovery}

In recent years, many researches paid attention to assistive technology. A number of robotic systems have been reported that support and assist human beings such as exoskeletons and prosthetic limbs. Most researches focus on supporting the human physical functions in terms of rehabilitation or healthcare. However, the human cognitive functions typified by facial or body expressions are as important as the physical functions.

By smiling a person can show affection, humor, and put others at ease, which are essential traits in natural human communication. The main objective of the "Smile Recovery" project is the design of a supportive device to recreate facial expressions and to put the "smile" back on the face. In general, the main noninvasive cure for facial paralysis is the use of physical therapy, although it is not able to support permanently paralyzed patients. Other standard treatment methods work together with invasive techniques to support permanently paralyzed patients. Although there is a possibility of using some other non conventional methods such as Functional Electrical Stimulus (FES), the technique is still in the experimental stage, and the effect also is only momentary (Dingguo and Kuanyi; 2004). On the other hand, Robot Assisted Smile Recovery investigates the support of facial expressiveness though the use of a robotic technology based wearable supportive device called The Robot Mask (Fig. 1). The four key features of this proposed design are:

1. Silent Actuation: a major characteristic of facial expressions is their silent nature of occurrence. Due to the mechanical noise present in traditional actuators such as electrical motor based actuators, it will be improper to use them for facial expression generation. In the Robot Mask this problem is solved by the use of specially designed Shape Memory Alloy (SMA) based actuators.

2. Use of bioelectrical signals from contralateral face to generate skin displacements in the ipsilateral face: This would help to reduce the facial disfigurement due to facial asymmetry. The use of bioelectrical signals of the mask wearer will facilitate the interpersonal timing of facial expressions associated with each individual.

3. Noninvasive: this will eliminate complications due to medical surgeries, reduce maintenance difficulties, and increase the use among both temporary and permanent facial paralysis.

4. Natural looking smile: based on a rigorous analysis of the facial morphology, artificial expressions that closely resemble natural expressions are recreated. Because of the importance and universality of facial expressions it is necessary for artificially reconstructed expressions to be as close to the natural ones as possible. 


\section{Analysis of Facial Morphology}

Even though in case of illness or accident, muscle movement can be lost, the functions of natural muscles can be recreated artificially. In the case of muscles on the face, artificial muscles can be attached externally to the surface of the face to generate artificial facial expressions. This section introduces an analysis of both amount and direction of the facial surface skin displacement by using markers on the face and 3-dimensional imaging. It will be shown how artificially generated smiles can be generated and made to look similar to natural ones.

\subsection{Preliminary experiments}

In order to be able to recreate a smile adequately, it is necessary to perform a systematic analysis of the facial skin displacement due to natural muscle contraction. At the moment the focus is in the displacement between the neutral and the smiling face.

In a preliminary experiment, it became obvious that some areas of the face are subject to greater displacement than others during smiling. Markers were placed on the face in order to be able to track the displacement more accurately. Especially the area around the mouth shows greater displacement, as can be seen in figure 2, which shows a healthy subject smiling on command.

In order to recreate the "smile" it was attempted to displace that area of greatest natural displacement artificially. Figure 3 shows an artificially generated facial expression. It was possible to verify that artificial facial expressions can be achieved by displacing the surface of the skin using point based actuation. Because of the skin elasticity the displacement achieved by the actuators is propagated, displacing the area surrounding it. The surface skin is displaced artificially but it is not yet a good facsimile of the "smile". Therefore, a more in-depth analysis of facial displacement is necessary in order to recreate the smile in a convincing manner.

\subsection{Systematic facial displacement analysis}

For the systematic facial displacement analysis and accurate measurement of the skin displacement, markers were attached to the subject's face as in the previous experiment. However new patterns of markers were introduced. The first pattern used was in the form of a grid and it used the natural landmarks of the face, such as nose, eyes and chin as reference points. The second pattern used was based on the muscles activated while smiling: a series of markers was attached over the lines of the muscles zygomaticus major, zygomaticus minor and risorius. The location of the muscles on the face can be seen in figure 4 .

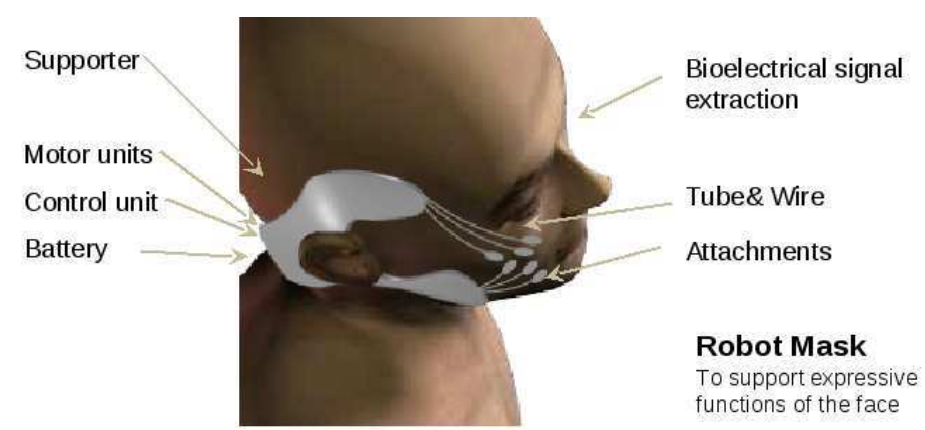

Fig. 1. The Non Invasive Robot Mask 


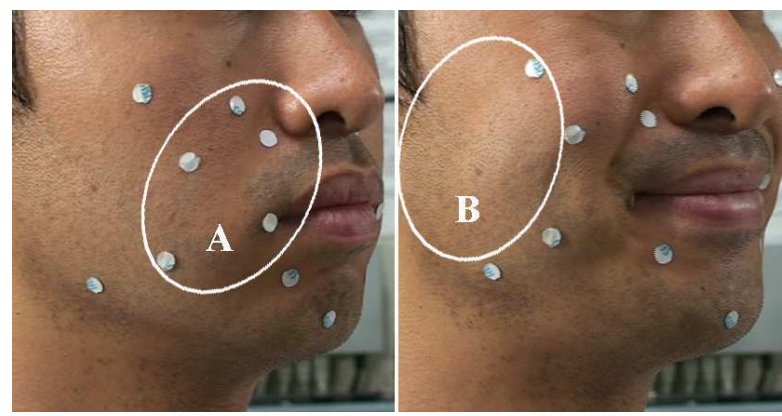

Fig. 2. Facial skin displacement in a healthy subject. The area designated with A shows the greatest displacement while smiling while B shows little displacement.

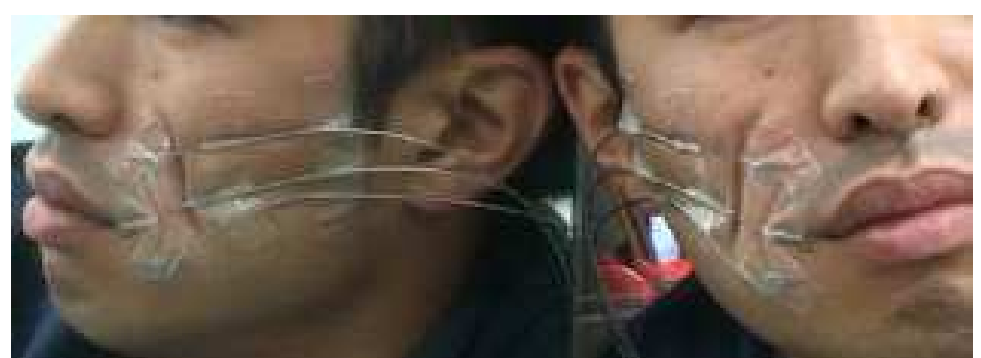

Fig. 3. Artificial facial skin displacement using 3 artificial muscles

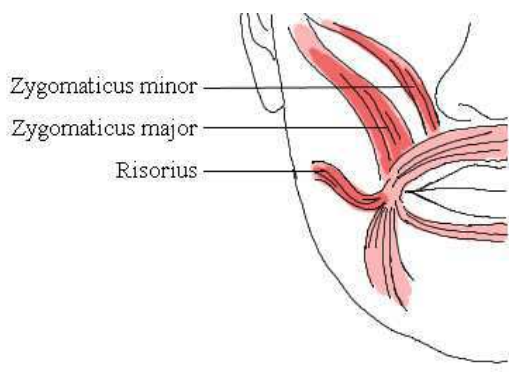

Fig. 4. Muscles on the face 

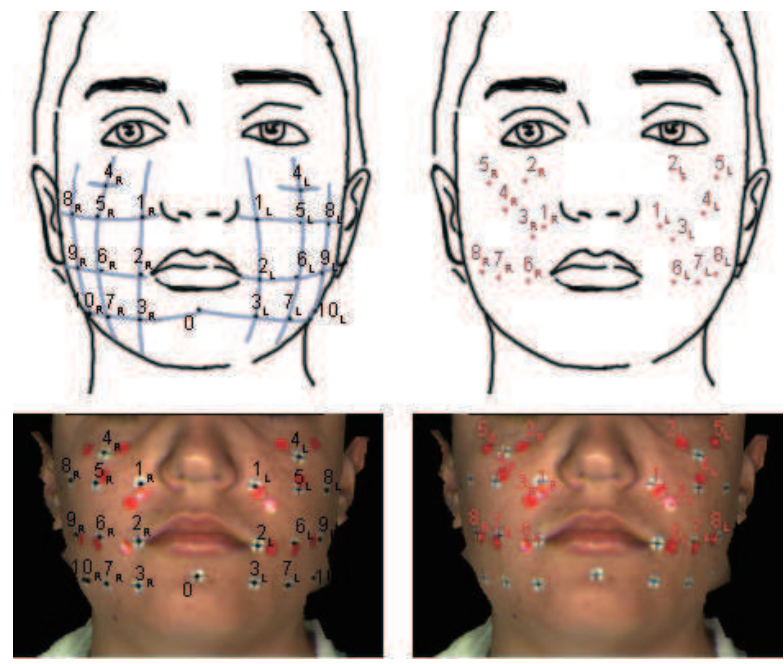

Fig. 5. Position of Markers on the face. Left: Grid-line markers. Right: Muscle markers

Figure 5 shows the position of both the grid-line and muscle markers. The grid-line markers are white with a back cross while the muscle markers are red. There are 10 grid-line markers on each side and one on the chin and 8 muscle markers, designated with the subindexes " $\mathrm{L}$ " and " $\mathrm{R}$ " for the left and right hand side respectively.

The subjects are photographed using a 3-dimensional imaging device. This serves the dual purpose of guaranteeing that the frontal and lateral views correspond to the same moment in time and makes rotation in 3 dimensions possible for calibration. After capture the images are rotated and scaled so that the eyes, ears and nose of both the neutral and smiling face match. Then, the frontal and lateral views were separated for analysis. These views can be seen in figure 6 .

In order to calculate the total 3-dimensional displacement the horizontal and lateral displacement was measured and the Pythagoras equation was used. The total displacement of the $\operatorname{dot}\left(D_{T}\right)$ is the square root of the sum of the squares of the frontal displacement $\left(D_{F}\right)$ and the lateral displacement $\left(D_{L}\right)$ of the dot (Equation 1).

$$
D_{T}=\left(D_{L}^{2}+D_{F}^{2}\right)^{1 / 2}
$$

The frontal displacements is calculated in a similar manner from the square root of the squares of the vertical displacement $(h)$ and the horizontal displacement $(l)$ of the dot in the frontal view (Equation 2).

$$
D_{F}=\left(h^{2}+l^{2}\right)^{1 / 2}
$$

In the first part of the experiment the subject was asked to show a neutral expression and then to smile.

Figure 7 shows the 3-dimensional linear displacement of both grid-line and muscle markers between the neutral face and a natural smiling face. In accordance with previous studies that state that facial asymmetry is substantially left-sided regardless of emotional valence (Borod 


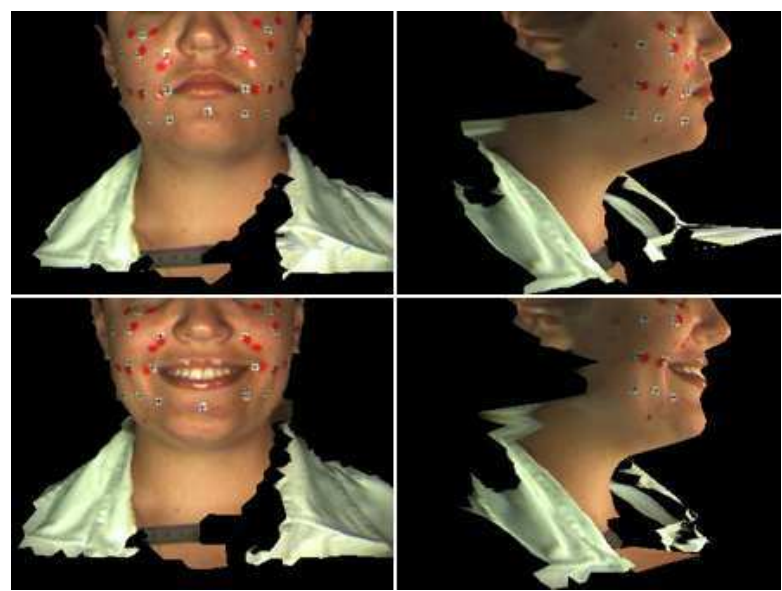

Fig. 6. Frontal and lateral views for neutral face (top) and smiling face (bottom)

et al.; 1998), a more pronounced displacement in the left side of the subject's face was observed.

By systematically analyzing the facial displacement between the neutral and the smiling face on a healthy subject it could be determined that the displacement on skin markers never exceeds $20 \mathrm{~mm}$ for the smile. It could also be noted that the greatest displacement correspond to grid-line points 2 and 6 which follow the horizontal line of the smile (Fig. 7), which is not surprising when the shape of the smile is considered.

In the second part of the experiment it was attempted to replicate the skin displacement during smiling using cables attached to the end points of the muscles. Cable 1 pulled from underneath the ear, simulating the muscle risorius, cable two pulled from above the ear simulating the muscle zygomaticus major. Three artificial expressions were created: artificial displacement by pulling cable 1 and cable 2 individually in turn and the facial displacement achieved by pulling both cables at once. 3-dimensional images were taken of the artificial facial expressions.

Figure 8 shows the displacement for artificially generated facial expressions for the artificial risorius simulator, artificial zygomaticus major simulator, and both simultaneously as well as the displacement during the natural smile for comparison. It should be noted that the artificial smiles were able to achieve their greatest displacement on the same points of greatest displacement as the natural smile (Fig. 8).

Also of interest when analyzing facial expressions, it is not only the amount of displacement that is important but also the direction towards which the displacement occurs. Studies have shown that angular deviations are more significant to the detection of facial symmetry than linear displacements (Rhodes et al.; 2005). The angle of displacement of both grid-line and muscle markers was analyzed and compared to the displacement during a natural smile. Figures 9 and 10 show the histograms for the difference in the displacement angles when compared with the natural smile for grid-line and muscle markers respectively. The values are given in degrees. 


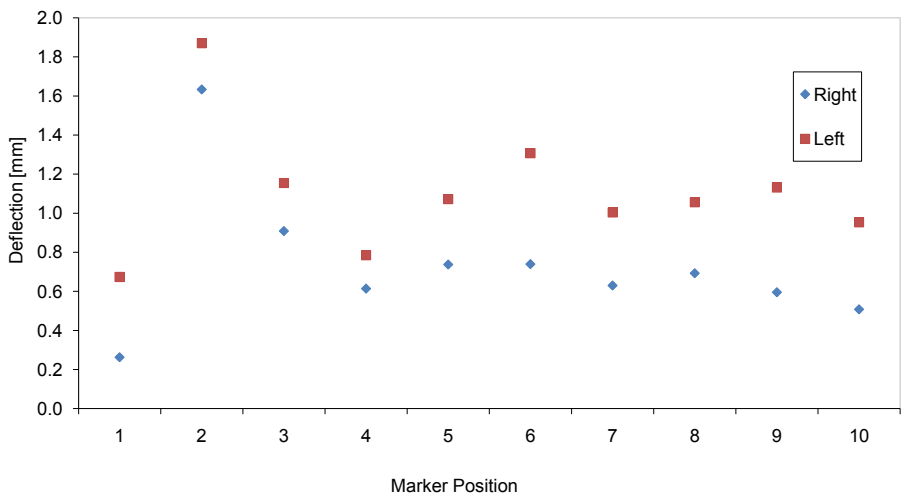

(a)

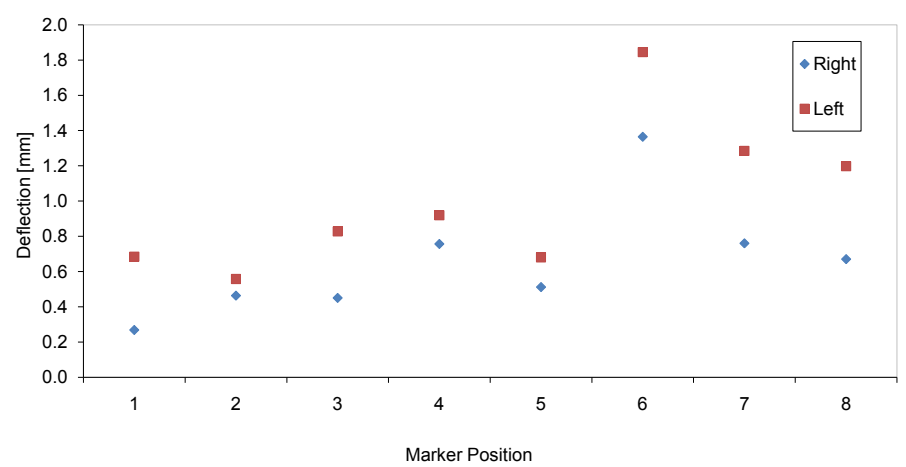

(b)

Fig. 7. 3-dimensional displacement [in $\mathrm{cm}$ ] of (a)Grid-line markers and (b)Muscle markers between the neutral and the naturally smiling face.

An example of the artificial facial displacement, using the artificial risorius simulator and artificial zygomaticus major simulator can be seen in figure 11. The symmetrical image is constructed using a hemifacial actuation image and its mirror image.

From the experiments in artificial displacement of the facial skin it could be seen that pulling just from underneath the ear led to an amount of displacement similar to that of the natural smile both in grid-line and muscle markers, the angle of displacement was however not similar enough as the mouth was pulled downwards and not enough up. When pulling above the ear a smaller displacement than that of the natural smile could be observed, the angle of displacement however was very similar. Especially around the points of greatest displacement (grid-line points 2 and 6), the difference in displacement angle was under $5.5^{\circ}$ which can be considered a good approximation of the smile. However the best approximation of the smile was achieved when using both artificial muscles simultaneously (Figures 9 and 10) which in accordance with the fact that in the healthy human being multiple muscles are used to create the smile. 


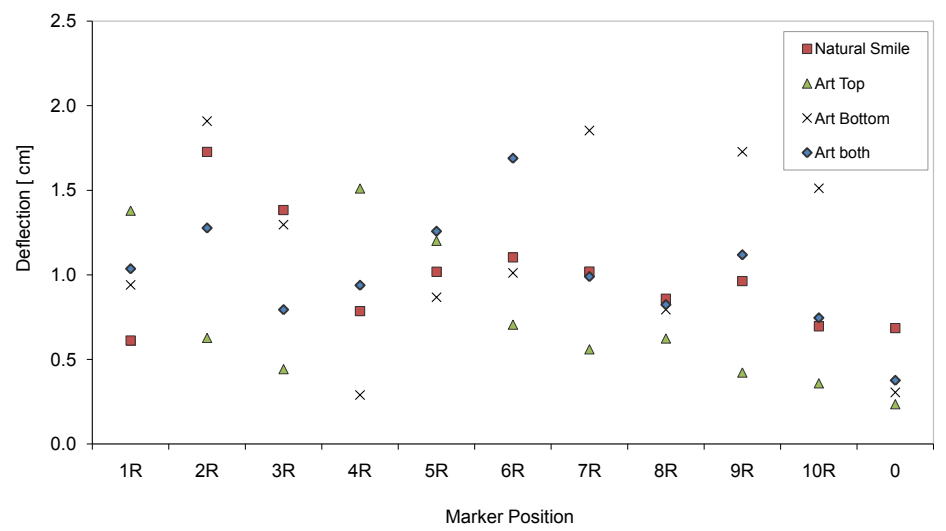

(a)

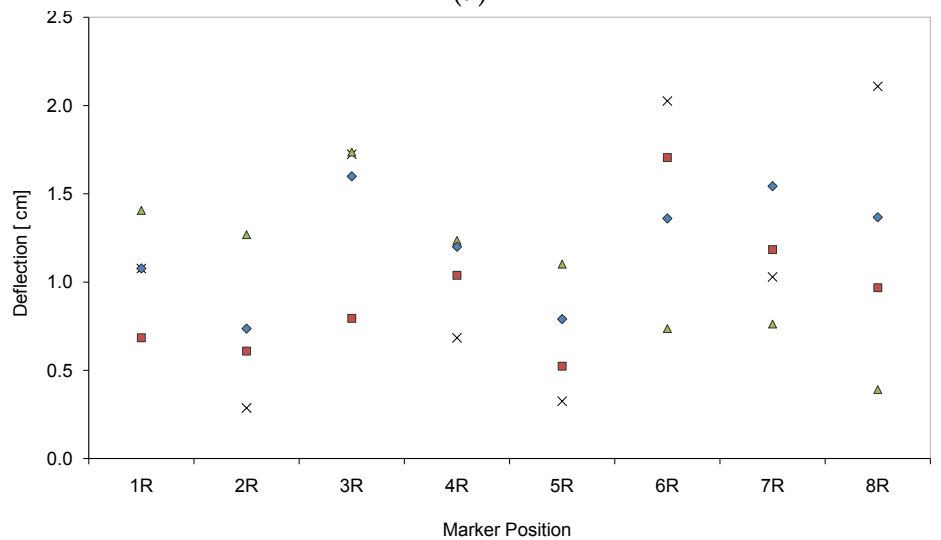

(b)

Fig. 8. 3-dimensional displacement [in $\mathrm{cm}$ ] of (a)Grid-line markers and (b)Muscle markers between the neutral and natural smile, artificial risorius simulator ("Art Bottom "), artificial zygomaticus major simulator ("Art Top "), and both simultaneously. 


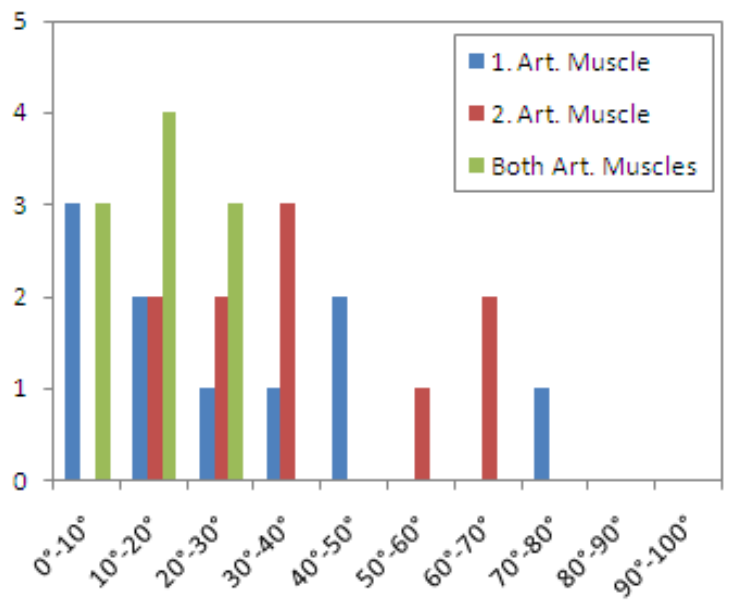

Fig. 9. Histogram of the error between the deviation angles of artificial and natural expressions for the artificial risorius simulator (1. Art. Muscle), artificial zygomaticus major simulator(2. Art. Muscle), and both simultaneously (Both Art. Muscles) of grid-line markers.

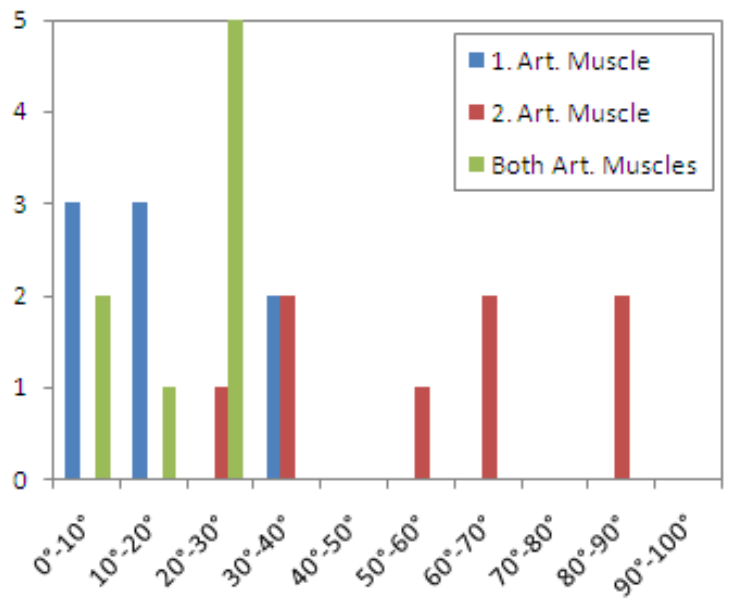

Fig. 10. Histogram of the error between the deviation angles of artificial and natural expressions for the artificial risorius simulator (1. Art. Muscle), artificial zygomaticus major simulator(2. Art. Muscle), and both simultaneously (Both Art. Muscles) for Muscle markers. 

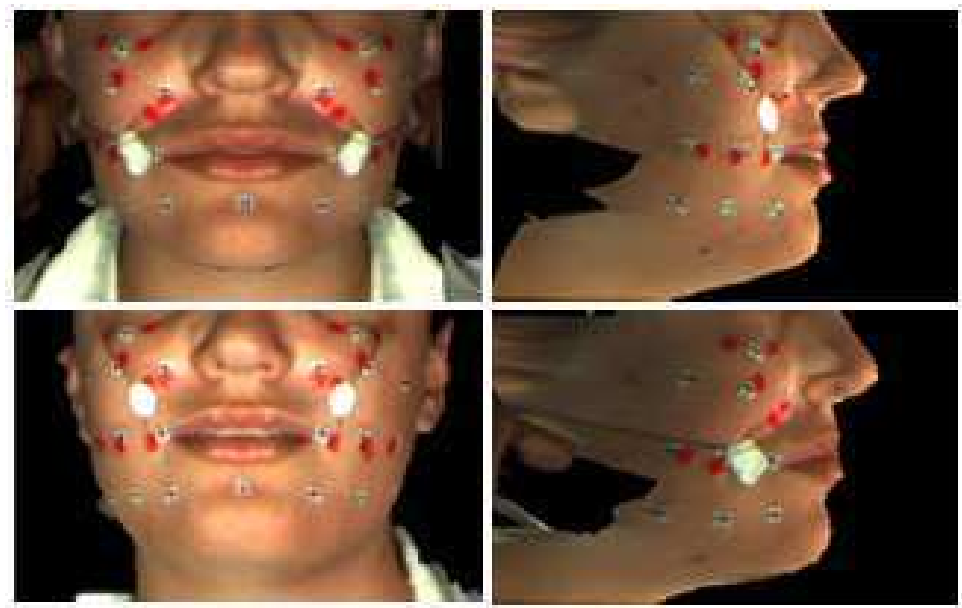

Fig. 11. Mirrored reconstruction of a hemifacial image where the smile is simulated using artificial risorius simulator (top), artificial zygomaticus major simulator (bottom)

Figures 7,8 and 11 show that it is possible to simulate a smile artificially by using artificial muscles.

The method for the systematic analysis of facial displacement that was introduced in this chapter using grid-line and muscle markers simultaneously and using 3-dimensional image capturing is well suited for future calibration of the Robot Mask for individual users. Further, in the case of subjects suffering from only hemi-facial paralysis, it can be used to acquire the displacement amount and angles from the healthy side as a template for the artificial displacement on the paralyzed side.

In order to further analyze the 3-dimensional facial surface skin displacement, the artificial smile and natural smile's frontal protrusions were compared to the neutral face. The results can be seen in figure 12. The right side of the subject's face was displaced using two artificial muscles simultaneously. It can be seen that the same areas are displaced as in the natural smile: around the mouth and cheek. Notably, there is little displacement occurring in both the natural and the artificially generated smile in the area around the eyes. This shows that the efforts for smile recovery should concentrate on the area around the mouth.

Taking into account these results, the Robot Mask was designed in order to artificially displace the facial skin.

\section{Robot Mask}

The Robot Mask, which consists of a head supporter, motor unit and a pulling wire arrangement, attaches onto the face externally and pulls facial skin through cables attached to the skin (Fig. 13). The pulling movement is derived by a specially designed SMA based actuator called "Silent Actuation Unit" or SIAC unit in short. The basic principle is the contraction of SMA coil elements when undergoing Joule heating. 


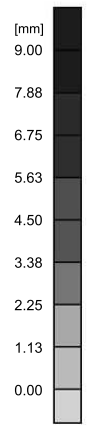

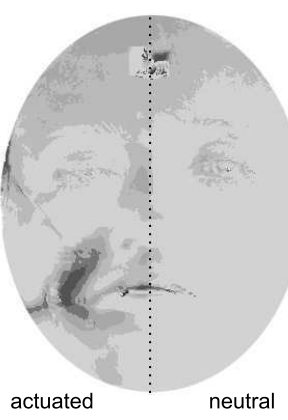

(a)

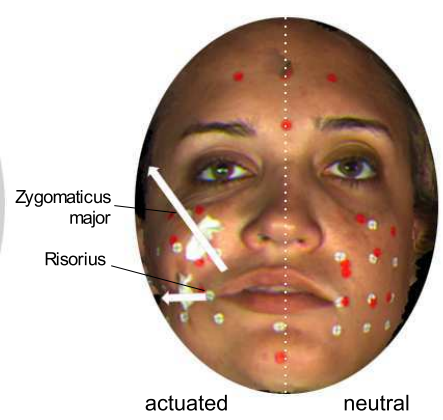

(b)

Fig. 12. Artificial facial displacement through actuation on the subject's right side. (a) Compared 3-dimensionally to the neutral face. (b)Actuation direction and corresponding muscles.
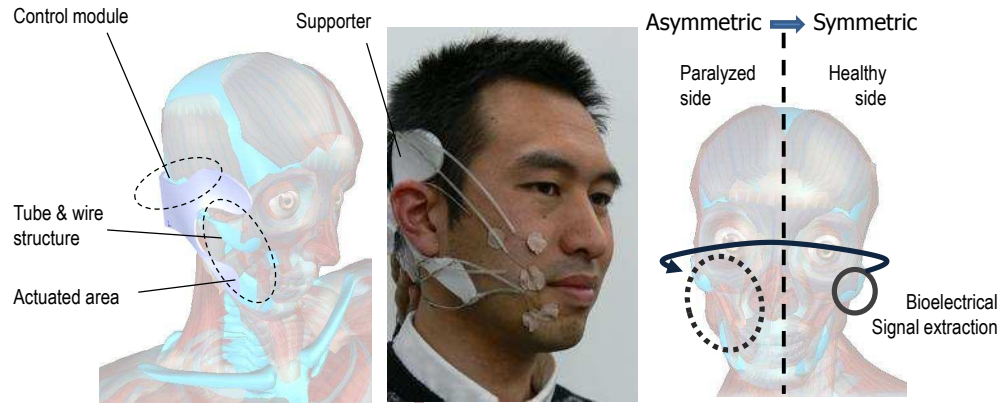

Fig. 13. The basic concept of Robot Mask 


\subsection{Shape Memory Alloys and Actuators}

The Shape Memory Alloy group, which was first investigated in 1951 through a goldcadmium alloy (Higgins; 1993) poses two distinct properties: the shape memory effect and the pseudo-elasticity. Pseudo-elastic materials show a phase transformation between Martensite and Austenite under mechanical loading and a complete reversal during subsequent reheating. Bellow a certain temperature (the critical temperature), a martensitic type of structure is formed with the alloy carrying a memory of the high and low temperature shapes. Furthermore, the superelastic characteristics of shape memory alloys (SMA) will make them capable of producing large recoverable strains (ASM; 1990).

There have been several reports, especially in recent times, about SMA based actuators. (Mascaro and Asada; 2003) reported on a wet shape memory alloy actuator which can be used for a robotic flesh. They kept the actuator immersed inside water and an electrical current was used to heat the SMA. They also proposed a hybrid electric/thermofluidic control method which uses water for cooling and either electric current or water for heating. Asada et al. (2006) presented a SMA actuator system for a 12 axis tendon actuator of a five fingered robot hand. (Yang and $\mathrm{Gu}$; 2008) introduced the concept of a planar bending embedded SMA actuator, which shows better characteristics in both heating and cooling. Rediniotis et al. (2002) proposed an SMA actuator system which uses Fuel as the energy source, with convection heating and cooling using water. (Yambe et al.; 2001) in Japan have proposed a series of biomedical applications of SMA actuators. In 2001 they reported on SMA for internal artificial organs. They include the circulatory assistance for a heart failure and an artificial sphincter. Another biomedical application of Ni-Ti SMA is to create compressive stresses that promote knitting of broken bones. Shape-memory $\mathrm{Ni}$-Ti alloys also have been employed experimentally to dilate blood vessels, thus increasing the flow of blood to vital organs (ASM; 1990).

\subsubsection{Mechanical Properties of Actuation elements}

Since it is possible to generate heat in an SMA coil though Joule heating by applying an electrical potential across it, several experiments were conducted to investigate its extension/contraction characteristics against different controlled actuation conditions. Figure 14 shows the performance of SMA coils under various such controlled conditions. These experiments were done using artificial muscles of initial length $20 \mathrm{~mm}$ and initial expanded length $45 \mathrm{~mm}$. Figure 14(a) shows the wire deflection with time for low actuation voltages. Three tests were done using actuation voltages 1.5, 1.75 and 2.0 and the characteristics were compared with the $4.0 \mathrm{~V}$ actuation. As can be seen from the graphs, the maximum actuation, as well as the actuation rate, show a directly proportional relationship to the actuation voltage. Figure 14(b) shows the time-displacement characteristics in heating and cooling. The graph shows a significant difference in performance during heating compared to cooling. Although deflection during heating is completed in 1.1 seconds, as many as 32 seconds were necessary for the cooling process to finish completely. Both the tests in figures 14(a) and 14(b) were carried out under no load conditions with natural drafting.

\subsection{Supporting Facial Expressiveness}

As indicated by Fig. 15, the Robot Mask has been designed to emulate a set of five muscles of the oral group of facial muscles, namely (1) Zygomaticus minor, (2) Zygomaticus major, (3) Risorius, (4) Platysma, and (5) Depressor Anguli Oris. Two actuators are been used to generate the movement of Zygomaticus major. 


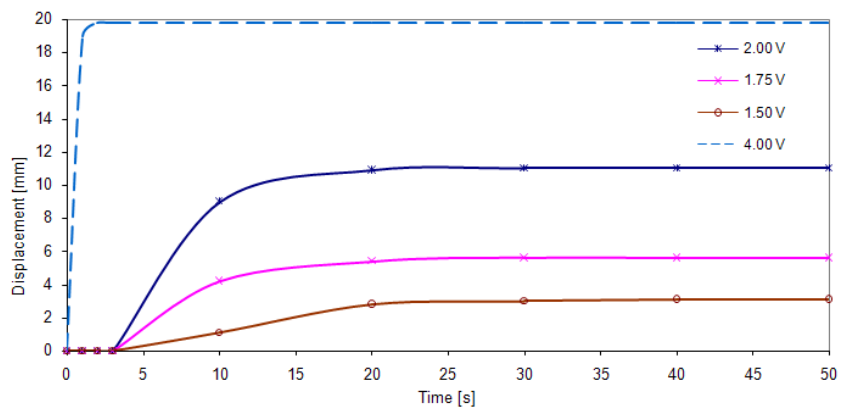

(a)

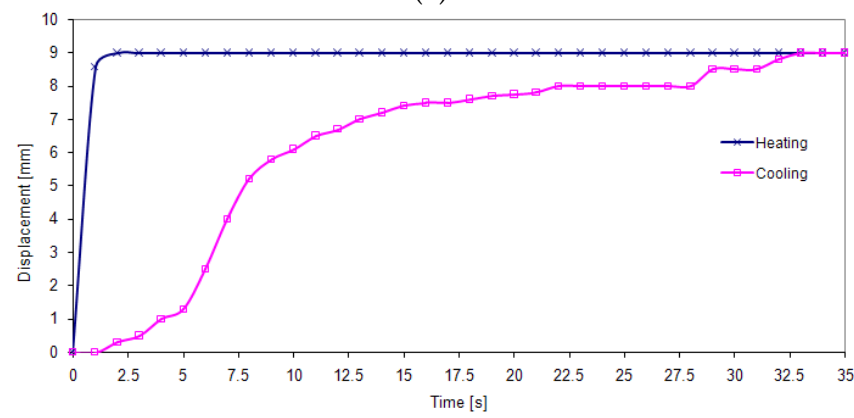

(b)

Fig. 14. Performance of SMA coils type actuation elements under various controlled conditions. (a) Low voltage actuation characteristics, and (b) Time-displacement characteristics during heating and cooling
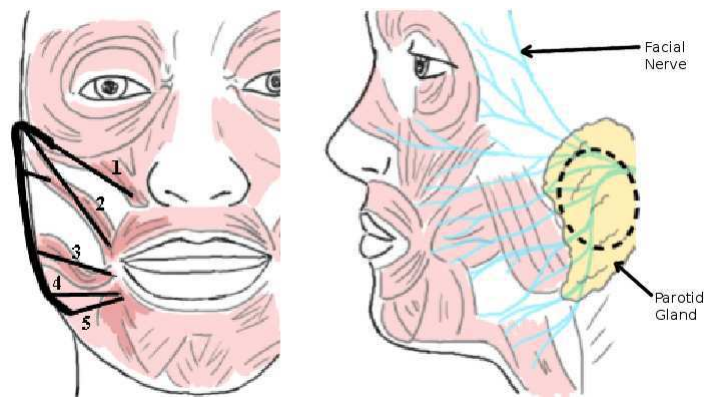

Fig. 15. Part of the oral group of facial muscles under consideration, actuator positioning, and the location of Parotid Gland with the emergence of the facial nerve highlighted with the dotted ellipse 


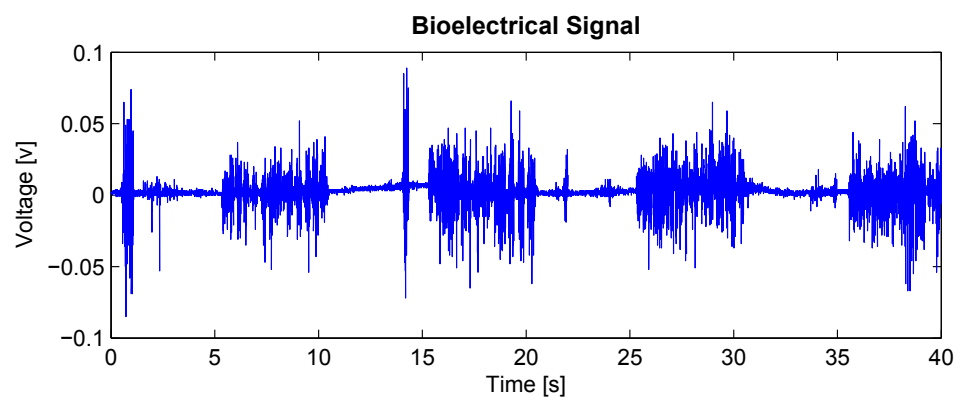

Actuation Signal

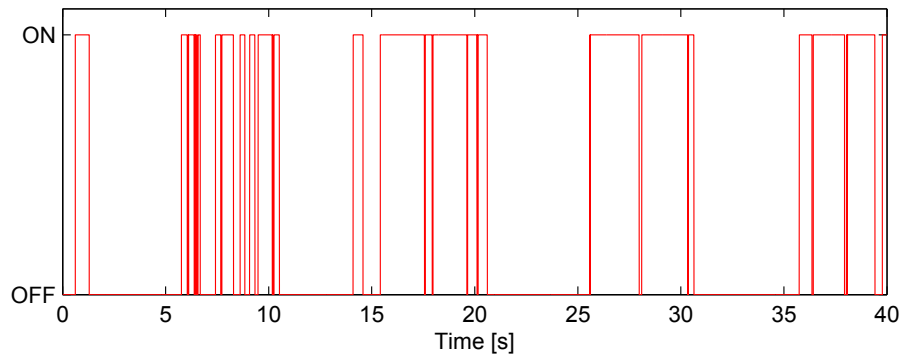

Fig. 16. Bioelectrical signal and the corresponding actuation signal. On top the raw signal and at the bottom the firing pattern

The voluntary responses of facial muscles are initiated from the appropriate regions of the brain, and the facial nerve helps to transmit these discharges to the facial muscles by using five terminal groups of branches, that emerge from the upper, anterior, and lower borders of the parotid gland, which is located below the lower half of the ear.

\subsubsection{Bioelectric signal based control}

The bioelectrical signals were extracted using $\mathrm{Ag} / \mathrm{AgCl}$ flat type active electrodes which were placed near the parotid gland (Fig. 15) with the electrodes being $20 \mathrm{~mm}$ apart. The third ground electrode was placed on the neck. Since the gel between the skin and the electrode takes some time to settle down, 15 min were left before taking any data. The signals were acquired through the 10bit ADC port of the master microcontroller at a sampling rate of $1 \mathrm{kHz}$. The sampled data was sent to a 50 size FIFO buffer and then the squared values of buffer data were averaged and compared against a predefined threshold value. In this way it was possible to identify the presence of any bioelectrical signal that is different from the average neutral condition, however no attempt was made to identify the individual muscles associated with the signal.

Figure 16 shows a time sequence (represented in terms of it's sample number) of bioelectrical signal captured at a rate of $200 \mathrm{~Hz}$ and the corresponding actuation logic derived by the master controller. The data corresponds to a time period of $40 \mathrm{~s}$. 


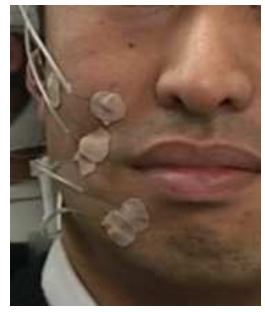

a

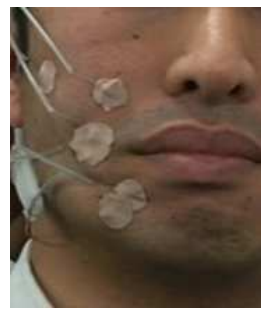

C

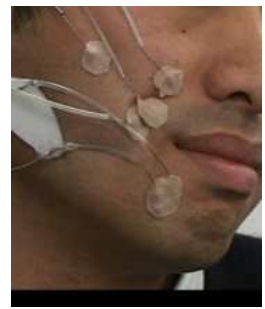

$\mathrm{b}$

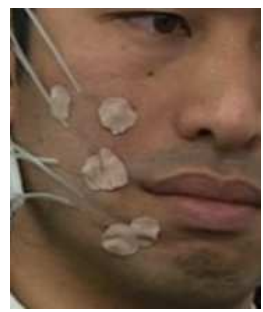

d

Fig. 17. Formation of artificial expressions through the Robot Mask.

\subsection{Evaluation of Robot Mask Performances}

The figure 17 shows three different sets of artificially generated facial actions. The tests were carried out using 6 artificial muscles in the arrangement explained in figure 15. Figures 17 (a) and (b) shows the front and side views of the effect of actuator 2 which is somewhat equivalent to the expression produced by the Zygomaticus major muscle. This resulted in a tiny wrinkle and a smile like pulling of a side of the mouth. Note that (a) and (b) are two different time instances and not the front and side views of same expression. 17(c) is the combined effect of actuators 4, 5 and 6 and Fig. 17(d) is the combined effect of all 6 actuators. In both those instances it is possible to see the circular type wrinkle around the mouth near the cheek. In (c) the wrinkle is more to the lower half of the face and in (d) it spreads fairly evenly around the mouth region. The pulling of wire 6 makes the back area of the facial skin move together with the area close to the mouth, making a larger deflection in skin with a less salient wrinkle around the mouth.

\section{Conclusion}

The two measures that influence the recreation of the natural smile identified by this research are the linear amount and the angle of facial skin displacement. As shown in this chapter, both were adequately emulated by the Robot Mask.

In this chapter it was shown that it is possible to generate artificial facial expressions that bear a close similarity to natural facial expressions by attaching cables to the surface skin of the face over the endpoints of the muscles zygomaticus major and risorius. In order to achieve an adequate artificial expression both in displacement amount and direction, it is necessary to use at least two cables.

The amount of facial displacement in a healthy subject, did not exceed the displacement range of the actuators, making the actuator based facial mask feasible. 
Because the individual artificial muscles used in the Robot Mask are capable of contracting up to $20 \mathrm{~mm}$ the feasibility of the Robot Mask has been further established.

The chapter also showed how SMA based soft actuators could be used effectively to pull facial skin and generate facial expressions artificially.

\section{Future works}

Enhancements to the Robot Assisted Smile Recovery project will be carried out along three directions:

1. Improvements to actuators: this will include point to point actuation control and improving actuation rate by employing forced SMA coolers

2. Extending to other areas of the face: will investigate on how the mask can be used to cover the whole face and emphasis will be given to Orbicularis muscles around eye and mouth

3. Aesthetic improvements: investigating on methods to attach wires to the face in a less visible manner

\section{References}

H. Wang and N. Ahuja. Facial expression decomposition. Proc. of Ninth IEEE International Conference on Computer Vision (ICCV), Volume 2:pp. 958-965, Oct. 2003.

M. Batty and M.J. Taylor. Early processing of the six basic facial emotional expressions. Cognitive Brain Research, 17(3):pp. 613-620, Oct. 2003.

G. Mandler, J. A. Russell, and J. M. Fernandez-Dols. The psychology of facial expression (studies in emotion and social interaction. Technical report, Cambridge University Press, 1997.

P. Ekman. Facial expressions of emotion: an old controversy and new findings. Philosophical Transactions of the Royal Society of London, 335:pp. 63-69, 1992.

P.J. Byrne. "importance of facial expression in facial nerve rehabilitation". Current Opinion in Otolaryngology and Head and Neck Surgery, Volume 12:332-335, 2004.

M. R. Garanhani, J.R. Cardoso, A. de Mello, G. Capelli, and M. C. Ribeiro. "physical therapy in peripheral facial paralysis: retrospective study". Brazilian Journal of Otorhinolaryngology, Volume 73(1):106-109, Jan/Feb 2007.

D.L. Beck and J.W. Hall. The hearing journal: Electroneuronography. Volume 54(3), March 2001.

F. B. Quinn and M.D. Jr. "facial nerve paralysis". [Online] Available: http://www . utmb. edu/otoref/grnds/face961.htm, March 1996.

Myles L. Pensak. Controversies in Otolaryngology. Thieme, first edition edition, 2001.

J. Harrigan, R. Rosenthal, and K. Scherer. The new handbook of methods in nonverbal behavior research. Oxford University Press, 2005.

M. Vaiman, N. Shlamkovich, A. Kessler, E. Eviatar, and S. Segal. Biofeedback training of nasal muscles using internal and external surface electromyography of the nose. American Journal of Otolaryngology, Volume 26(Issue 5):pp. 302-307, Sep. 2005.

K. Sugimoto1, T. C. Theoharides, D. Kempuraj, and P. Conti. Response of spinal myoclonus to a combination therapy of autogenic training and biofeedback. BioPsychoSocial Medicine, 1(18):18, Oct. 2007. [Online] Available: http://www.bpsmedicine. $\mathrm{com} /$ content/1/1/18. 
Mark May and Barry M. Schaitkin. Facial Paralysis: Rehabilitation Techniques. Thieme, 2003.

Z. Dingguo and Z. Kuanyi. "neural network control for leg rhythmic movements via functional electrical stimulation". Proc. of IEEE International Joint Conference on Neural Networks, Volume 2:1245-1248, 2004.

J.C. Borod, E. Koff, S. Yecker, C. Santschi, and J.M. Schmidt. Facial asymmetry during emotional expression: Gender, valence, and measurement technique. Neuropsychologia, Elsevier Science Ltd., Volume 36(Issue 11):1209-1215, November 1998.

G. Rhodes, M. Peters, K. Lee, M.C. Morrone, and D. Burr. Higher-level mechanisms detect facial symmetry. Proc. of Biol. Sci., 272(1570):1379-1384, July 2005.

R.A. Higgins. Engineering Metallurgy, volume Part 1: Applied Physical Metallurgy. Butterworth-Heinemann, 6 edition edition, 1993. pp. 402-403.

ASM Handbook: Properties and Selection: Nonferrous Alloys and Special-Purpose Materials, volume 2. ASM International, 10th edition edition, Nov. 1990.

S. A. Mascaro and H. H. Asada. Wet shape memory alloy actuators for active vasculated robotic flesh. Proc. of ICRA '03, IEEE International Conference on Robotics and Automation, Volume 1:pp. 282-287, Sep. 2003.

K. Yang and C.L. Gu. Modelling, simulation and experiments of novel planar bendign embedded sma actuators. Elsevier, Mechatronics(18):pp. 323-329, 2008.

T. Yambe, S. Amae, S. Maruyama, Y. Luo, H. Takagi, S. Nanka, A. Tanaka, N. Kamiyama, R. Ohi, K. Tabayashi, H. Takeda, M. Yamada, and S. Nitta. Application of a shape memory alloy for internal artificial organs. J Artif organs, 4(2):88-91, June 2001. The Japanese Society for Artificial Organs. 


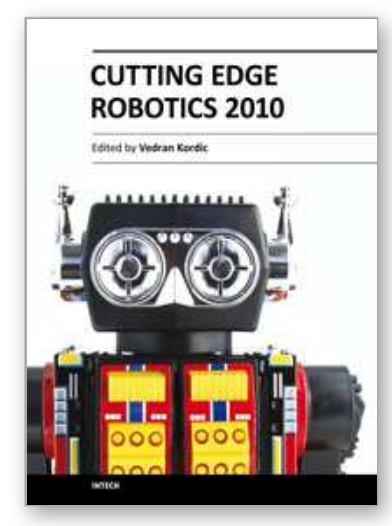

\author{
Cutting Edge Robotics 2010 \\ Edited by Vedran Kordic
}

ISBN 978-953-307-062-9

Hard cover, 440 pages

Publisher InTech

Published online 01, September, 2010

Published in print edition September, 2010

Robotics research, especially mobile robotics is a young field. Its roots include many engineering and scientific disciplines from mechanical, electrical and electronics engineering to computer, cognitive and social sciences. Each of this parent fields is exciting in its own way and has its share in different books. This book is a result of inspirations and contributions from many researchers worldwide. It presents a collection of a wide range of research results in robotics scientific community. We hope you will enjoy reading the book as much as we have enjoyed bringing it together for you.

\title{
How to reference
}

In order to correctly reference this scholarly work, feel free to copy and paste the following:

Dushyantha Jayatilake, Anna Gruebler and Kenji Suzuki (2010). Robot Assisted Smile Recovery, Cutting Edge Robotics 2010, Vedran Kordic (Ed.), ISBN: 978-953-307-062-9, InTech, Available from:

http://www.intechopen.com/books/cutting-edge-robotics-2010/robot-assisted-smile-recovery

\section{INTECH}

open science | open minds

\section{InTech Europe}

University Campus STeP Ri

Slavka Krautzeka 83/A

51000 Rijeka, Croatia

Phone: +385 (51) 770447

Fax: +385 (51) 686166

www.intechopen.com

\section{InTech China}

Unit 405, Office Block, Hotel Equatorial Shanghai

No.65, Yan An Road (West), Shanghai, 200040, China

中国上海市延安西路65号上海国际贵都大饭店办公楼 405 单元

Phone: +86-21-62489820

Fax: $+86-21-62489821$ 
(C) 2010 The Author(s). Licensee IntechOpen. This chapter is distributed under the terms of the Creative Commons Attribution-NonCommercialShareAlike-3.0 License, which permits use, distribution and reproduction for non-commercial purposes, provided the original is properly cited and derivative works building on this content are distributed under the same license. 